\title{
Painful Pathologic Fracture of the Humerus: Percutaneous Osteoplasty With Bone Marrow Nails Under Hybrid Computed Tomography and Fluoroscopic Guidance
}

\author{
Giovanni Carlo Anselmetti, MD, Antonio Manca, MD, Gabriele Chiara, MD, \\ Sean Tutton, MD, Gabriella lussich, MD, GianCarlo Gino, MD, \\ Giovanni Grignani, MD, Cinzia Ortega, MD, Nora Moselli, MD, and \\ Daniele Regge, MD
}

\begin{abstract}
A case of a 75-year-old patient with a painful pathologic humeral shaft fracture, with unacceptably high surgical risk and unsatisfactory analgesia is reported. In this case, impaired arm function and persistent pain with conservative management resulted in a poor quality of life. Palliation with image-guided percutaneous osteoplasty was considered. Because of potential cement leakage, inadequate fracture reduction, the site of the fracture, and the mobility of the joints in that area, image-guided percutaneous delivery of metallic bone marrow nails implanted together with polymethyl methacrylate (PMMA) osteoplasty was performed. This procedure achieved humeral shaft stabilization, bone fragment alignment, fracture reduction, and pain relief.
\end{abstract}

\section{ABBREVIATION}

PMMA = polymethyl methacrylate

Orthopedic reduction and fixation is the standard treatment in most cases for painful pathologic fractures of long bones (ie, humerus and femur) secondary to metastatic bone tumors. A pathologic fracture of the humeral neck or shaft is commonly treated surgically employing nails, rods, or plate and screws $(1,2)$ combined with immobilization using an external brace support (3). Early stabilization has been associated with reduced mortality. Some patients are treated conservatively because of their poor clinical condition and comorbidities (ie, metastatic and cardiopulmonary disease)

\footnotetext{
From the Interventional Radiology Unit (G.C.A., A.M., G.C., G.I.), Oncology Unit (G.G., C.O.), Anesthesiology Unit (N.M.), and Radiology Unit (D.R.), Institute for Cancer Research and Treatment, Strada Provinciale 135, Km 3.95, 10060 Candiolo, Turin, Italy; Division of Vascular and Interventional Radiology (S.T.), Medical College of Wisconsin, Milwaukee, Wisconsin; and Orthopedic Oncology Unit (G.-C.G.), Hospital CTO, Turin, Italy. Received November 8, 2010; final revision received February 6, 2011; accepted February 11, 2011. Address correspondence to G.C.A.; E-mail: giovanni. anselmetti@ircc.it
}

None of the authors have identified a conflict of interest.

(c) SIR, 2011

J Vasc Interv Radiol 2011; 22:1031-1034

DOI: $10.1016 /$ j.jvir.2011.02.021
$(4,5)$. Conservative treatment, usually comprising only an external cast, leaves approximately one-third of patients with inadequate pain control $(6,7)$. In such cases, percutaneous osteoplasty (also called cementoplasty) could be performed for stabilization of the fracture. This procedure was previously described $(8,9)$, but results were discouraging because a high rate of delayed fractures of the treated bone shaft was reported. Previously, a case of a pathologic fracture of the humeral shaft was treated by percutaneous osteoplasty with use of a bone cement-filled catheter resulting in pain relief (10). However, the bone reinforced by the catheter filled with polymethyl methacrylate (PMMA) fractured at the site of the previous pathologic fracture within 3 months of treatment.

An experimental study using swine models (11) described the successful injection of bone cement into a covered metallic stent placed within long bones, and another study showed that the strength of a cement-filled stent is superior to osteoplasty alone, but the bending power and bending strength were unsatisfactory (12). We describe a case of a metastatic fracture of the humeral neck treated by percutaneous insertion of metallic nails through an 8-gauge cannula followed by PMMA bone cement injection. After 


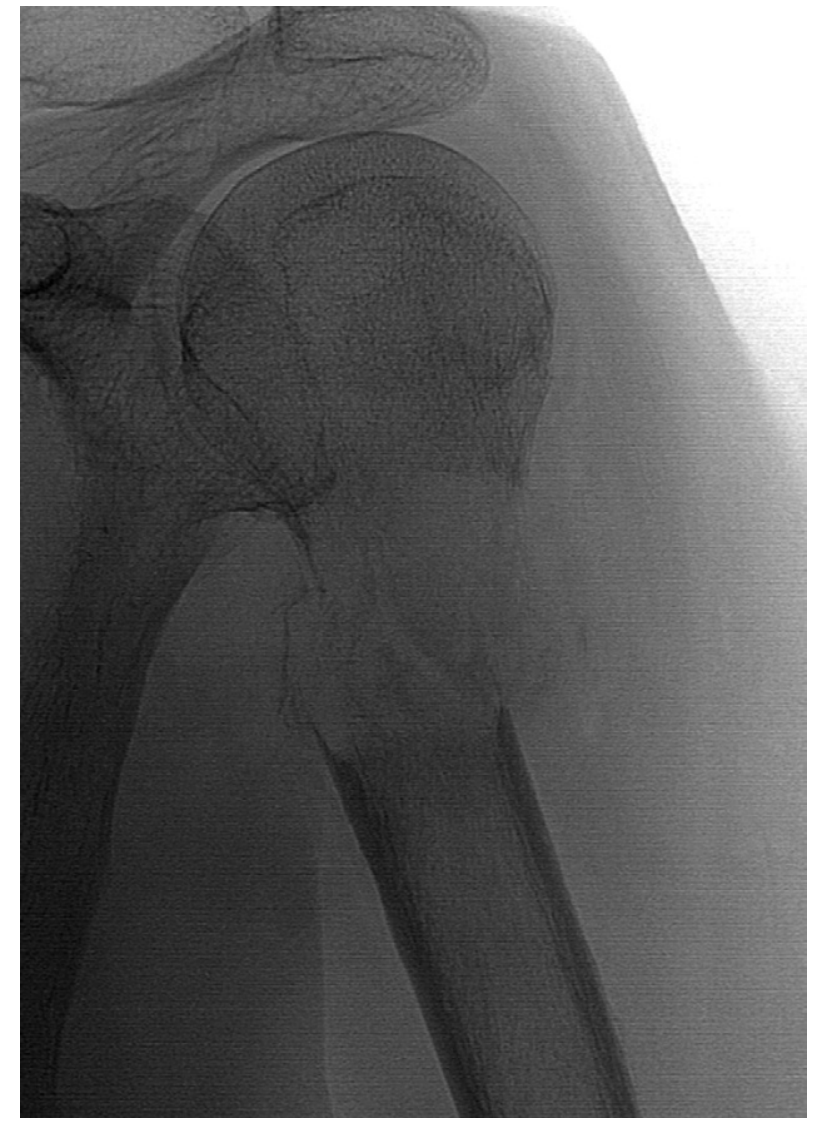

Figure 1. A plain radiograph shows an osteolytic lesion of the upper diaphysis of the left humerus with fracture displacement.

the procedure, the patient gained full restoration of functionality and pain relief without need for further analgesic therapy.

\section{CASE REPORT}

A 75-year-old man with multiple bone metastases from prostate cancer was referred for the treatment of a symptomatic (biopsy-proven) osteolytic metastasis of the left humeral neck. Imaging studies also showed an unstable fracture of the bone. Previously, the patient had undergone a surgical pelvic dissection for recurrence of carcinoma (lymphadenectomy, colostomy, and cutaneous ureterostomy were performed) and was bedridden because of massive bilateral deep vein thrombosis requiring an inferior vena cava filter. Pelvic abscesses, treated with percutaneous drainage, and a secondary enterocutaneous fistula complicated surgery. Surgical humeral stabilization was contraindicated because of comorbidities and the high risk of osteomyelitis related to pelvic abscesses.

Plain radiographs and spiral computed tomography (CT) with two-dimensional reconstruction showed an osteolytic lesion of the upper diaphysis of the left humerus with fracture displacement (Figure 1). The patient continued to have severe pain not controlled by intravenous narcotics and poor quality of life. Percutaneous treatment using osteoplasty combined with metallic nail insertion for stabilization was offered to the patient. After having been informed of the risks, the nonstandard nature of the treatment, and the benefits of this innovative intervention, and the patient signed the informed consent.

Immediately before the procedure, the patient was premedicated with intravenous antibiotics $(1 \mathrm{~g}$ of vancomycin hydrochloride and $100 \mathrm{mg}$ of gentamicin). The patient was placed in the supine position on the cradle of the angiographic suite, and the procedure was performed with digital hybrid fluoroscopic and CT guidance (Allura Xper CT, Philips, the Netherlands). Deep sedation (intravenous propofol, $200 \mathrm{mg}$, and fentanyl, $0.1 \mathrm{mg}$ ) was achieved, and continuous monitoring of vital sign parameters (electrocardiogram, blood pressure, oxygen saturation) was performed.

The percutaneous approach was performed with a 13gauge beveled vertebroplasty needle (OptiMed, Ettlingen, Germany) manually inserted through the humeral head. A 16 gauge Tru-Cut biopsy (Magnum; C.R. Bard, Murray Hill, New Jersey) was performed coaxially. The 13-gauge needle was advanced along the bone shaft to reach the distal part of the diaphysis (Figure 2). The correct needle pathway and its precise intraosseous positioning were assessed by rotational acquisitions with multiplanar reconstructions (Figure 3, a and b). A 13-gauge vertebroplasty needle was exchanged for a bigger 8-gauge metallic kyphoplasty introducer (Xpander Osteointroducer; Medtronic, Minneapolis, Minnesota) over a Kirschner guide wire. Through the 8 -gauge cannula, eight metallic tunnelers, normally used for central venous catheter placement, were used as bone nails (Bard Access Systems, Inc, Salt Lake City, Utah) (five tunnelers $2.6 \mathrm{~mm}$ diameter and three tunnelers $1.8 \mathrm{~mm}$ diameter; all $240 \mathrm{~mm}$ long) (Figure 4). The tunneler "nails" were sequentially introduced into the bone shaft and across the lesion by deploying them with the introducer stylet. This process was continued until satisfactory fracture alignment and shaft stabilization were obtained (Figure 5). Finally, a 10-gauge vertebroplasty needle was reinserted in the humeral shaft over the last metallic guide wire, and 20 $\mathrm{mL}$ of radiopaque bone cement (KyphX HRV, Medtronic, Minneapolis, Minnesota) was injected from distal to proximal to obtain complete filling of the humeral diaphysis, humeral head, and lesion (Figure 6, a and b). A CT scan with multiplanar reconstructions obtained after the procedure showed satisfactory bone fragment alignment and good lesion filling with minimal cement leakage at the metadiaphyseal fracture site surrounding the previously introduced nails.

The procedure was successfully concluded without any clinical complications. Procedural time was 1 hour. The patient reported complete pain relief within 24 hours, and he regained complete left arm functionality. Intravenous narcotics were progressively suspended within 1 week. During the 5-month follow-up, we observed stable pain regression and full arm functionality; during this time, the patient did not need to resume pain medications. 


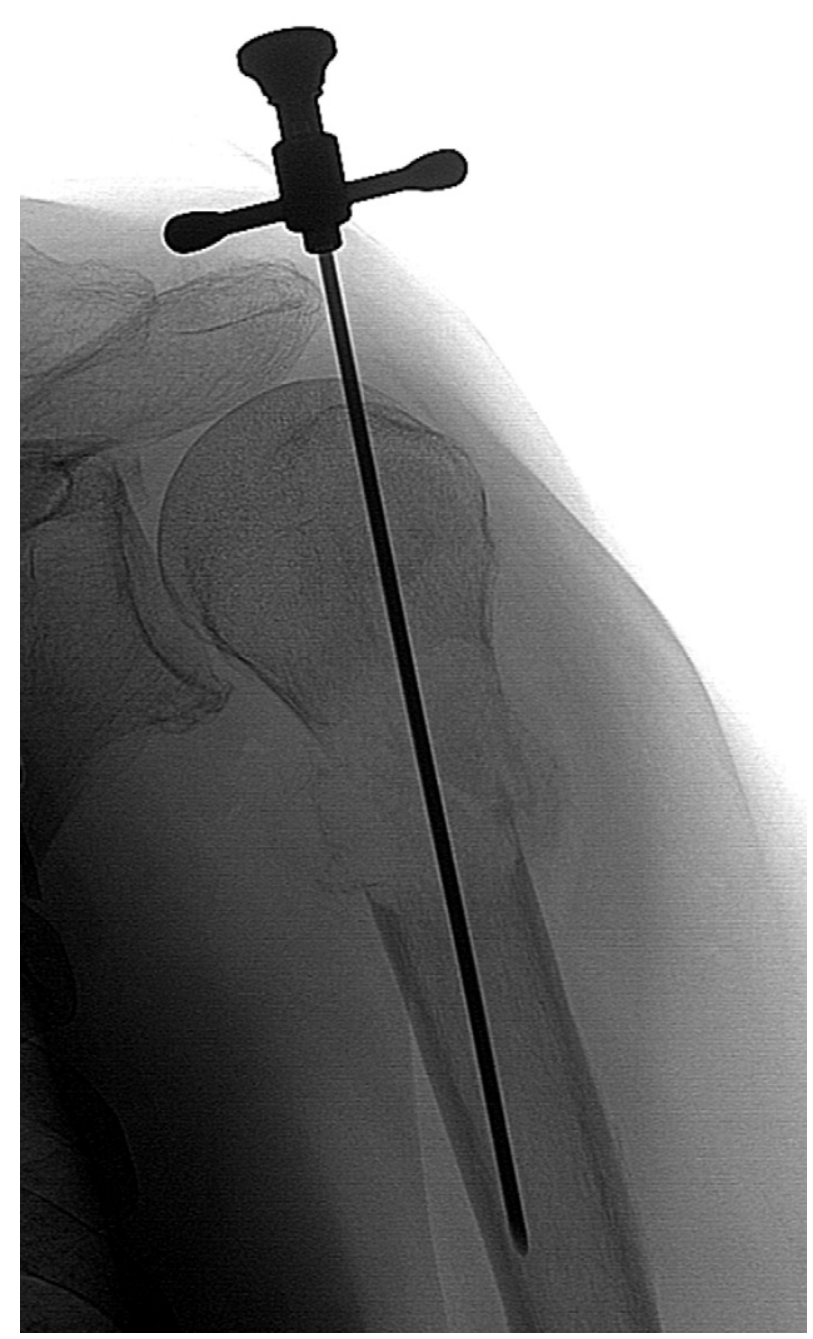

Figure 2. A percutaneous approach was accomplished with a 13-gauge beveled vertebroplasty needle inserted through the humeral head.

\section{DISCUSSION}

In the above-mentioned series $(8,9)$, osteoplasty was shown to be safe and effective in the treatment of painful bone metastases refractory to conventional therapy and with no surgical options. Although the complication rate was very low, subsequent recurrence of fractures in long bones suggested that the treatment was inadequate in those cases. Palliative care of patients with no surgical options is challenging considering that pain and poor quality of life can reduce a patient's life span. In the reported case, the diffuse cortical bone involvement could not be treated with standalone osteoplasty because of the high risk of bone cement leakage in the soft tissues and the unlikelihood of fracture stabilization. A similar pathologic fracture in the humerus shaft was previously treated with a cement-filled catheter (10), but, despite stable pain relief, the humeral fracture occurred again within 3 months. Two recent published experimental studies $(11,12)$ showed the possibility of achieving fracture stabilization with some kind of metallic device able to contain

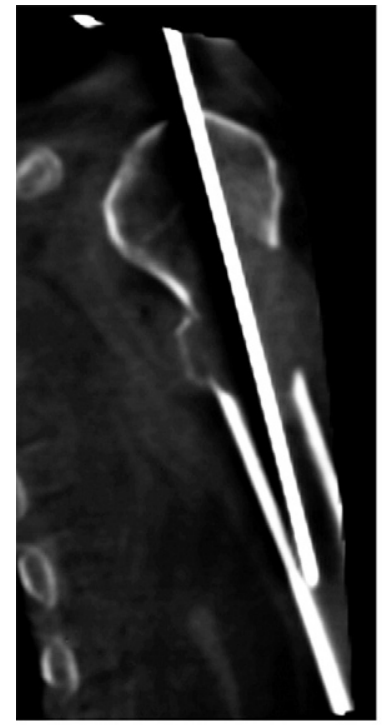

a.

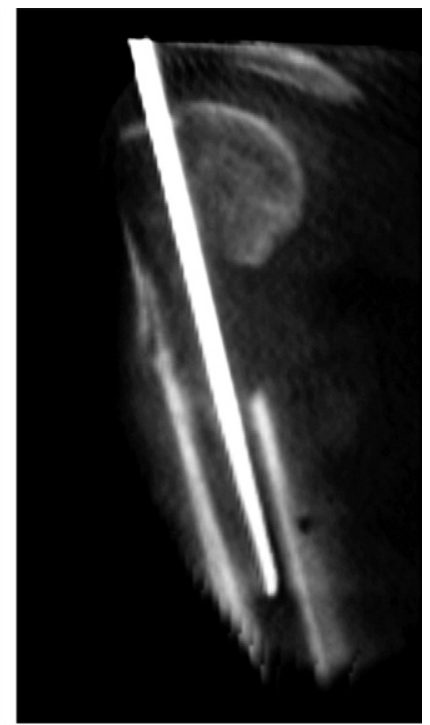

b.
Figure 3. The correct needle pathway and its precise intraosseous positioning were assessed by rotational acquisitions with coronal (a) and sagittal (b) multiplanar reconstructions.

Figure 4. Metallic tunnelers $(2.6 \mathrm{~mm}$ and $1.8 \mathrm{~mm}$ diameter, 240 $\mathrm{mm}$ long), normally used for central venous catheter placement, were used as bone marrow nails.

the injected cement. In the above-mentioned article $(11,12)$, the authors used a metallic endovascular stent that is very dissimilar to orthopedic surgical hardware and, in our opinion, suboptimal for stabilization of human long bones owing to its intrinsic flexibility.

We chose to insert metallic nails progressively and encase them in bone cement, mimicking an orthopedic surgical nail or rod stabilization. In our opinion, bone cement injection was useful to surround and stabilize the metallic nails and to fill the lesion achieving its partial necrosis (chemical and thermal). Hybrid digital fluoroscopic and CT guidance were essential to secure precise safe needle and guide wire insertion, avoiding displacement outside the bone. Rotational acquisition with two-dimensional reconstruction allowed precise targeting of the lesion reaching the distal part of the humeral shaft. Digital fluoroscopic monitoring during metallic nail insertion and bone cement injection was essential to avoid complications (ie, leakage) and to assess optimal lesion and bone filling. Early detection of a small cement leak in the humeral head resulted in immediate suspension of the injection avoiding endoarticular diffusion. The procedure was performed using deep intravenous sedation, lowering the anesthetic risks of standard surgical reduction. Full functional recovery occurred within 24 hours, with improvement in quality of life, opioid suspension, and early mobilization. Early reduction and mobilization has 


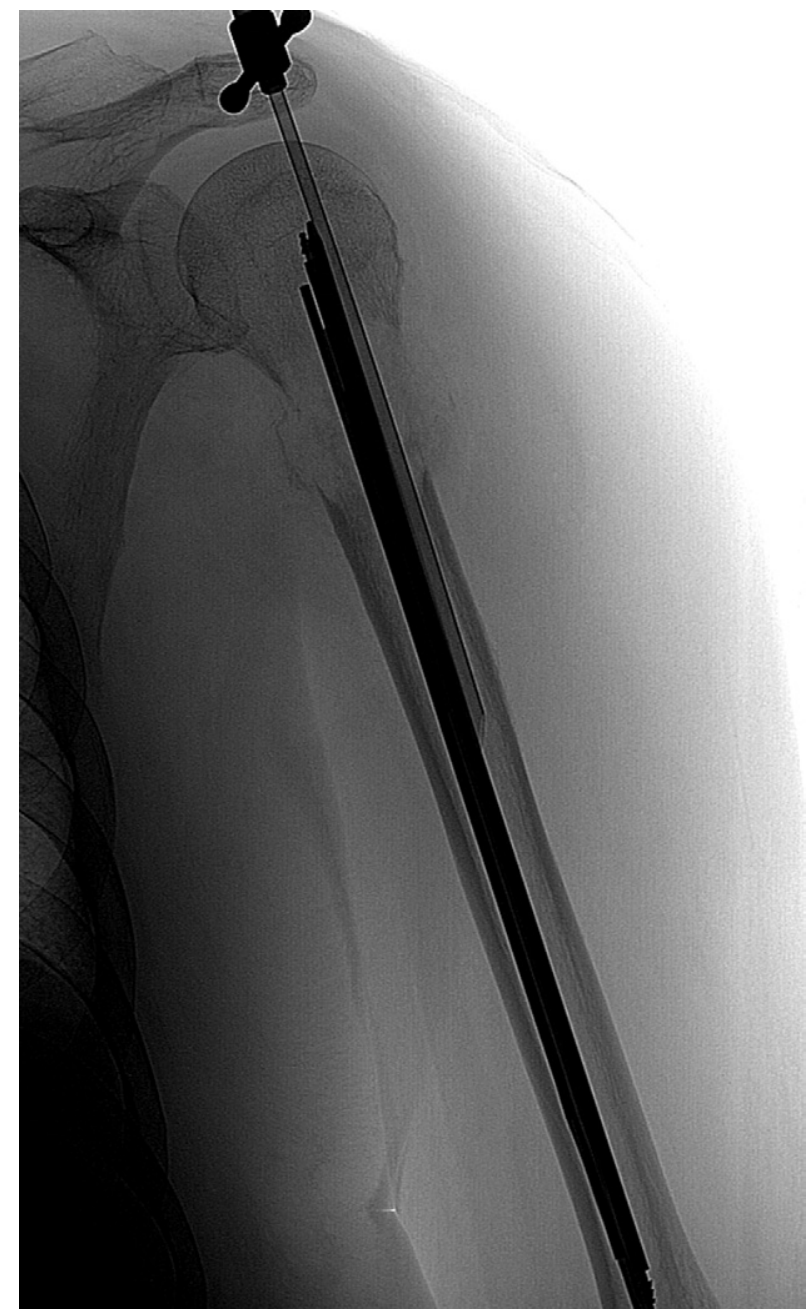

Figure 5. After a satisfactory fracture alignment was obtained, the vertebroplasty needle was reinserted for injection of bone cement.

been shown to be an important variable in reducing mortality in elderly patients. All of these factors are crucial in patients with cancer undergoing chemotherapy. Although the procedure was performed in an angiographic room instead of an operating room, sterile conditions were adopted, and our patient experienced no infectious complications during follow-up.

This case report shows that percutaneous stabilization of a humeral neck metastatic fracture is a safe and feasible treatment. Although surgery remains the first option, this case describes a new option of minimally invasive treatment for patients with a painful unstable pathologic fracture of the humeral shaft in which surgery is not feasible and pain is uncontrolled by conservative treatment. The exact procedure including size, length, and number of nails may vary depending on a patient's clinical history, location, and type of lesion. We believe that hybrid digital fluoroscopic and CT guidance offer a superior advantage to obtain precise needle insertion, metallic nail insertion, and cement injection.

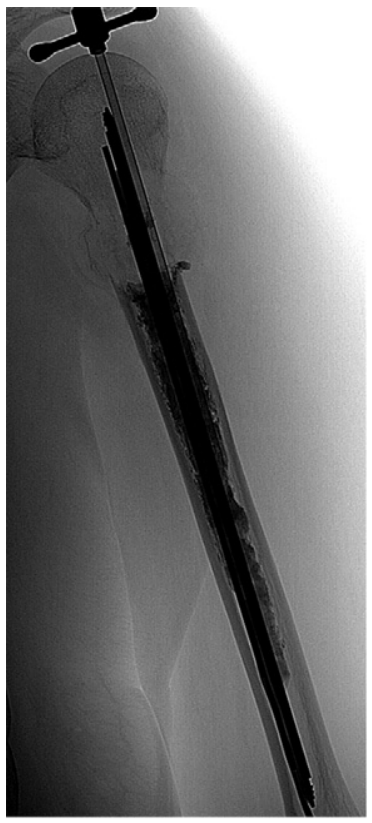

a.

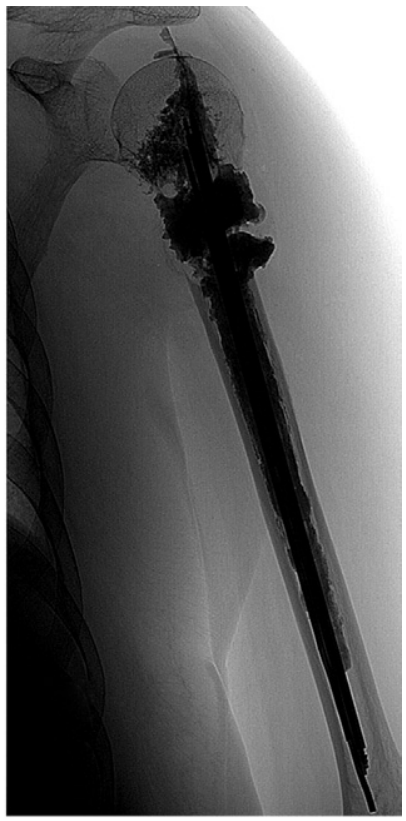

b.
Figure 6. The radiopaque bone cement was progressively injected following a distal-to-proximal pathway (a) to obtain complete filling of the humeral diaphysis, humeral head, and lesion (b).

\section{REFERENCES}

1. Dijkstra S, Stapert J, Boxma H, Wiggers T. Treatment of pathological fractures of the humeral shaft due to bone metastases: a comparison of intramedullary locking nail and plate osteosynthesis with adjunctive bone cement. Eur J Surg Oncol 1996; 22:621-626.

2. Bauze AJ, Clayer MT. Treatment of pathological fractures of the humerus with a locked intramedullary nail. J Orthop Surg (Hong Kong) 2003; 11:34-37.

3. Persad IJ, Kommu S. U cast or functional bracing following fractures of the shaft of humerus. Emerg Med J 2007; 24:361.

4. Perez CA, Bradfield JS, Morgan HC. Management of pathologic fractures. Cancer 1972; 29:684-693.

5. Krebs H. Management of pathologic fractures of long bones in malignant disease. Arch Orthop Trauma Surg 1978; 92:133-137

6. Meuser T, Pietruck C, Radbruch L, Stute P, Lehmann KA, Grond S. Symptoms during cancer pain treatment following WHO guidelines: a longitudinal follow-up study of symptom prevalence, severity and etiology. Pain 2001; 93:247-257.

7. Urch C. The pathophysiology of cancer-induced bone pain: current understanding. Palliat Med 2004; 18:267-274.

8. Anselmetti GC, Manca A, Ortega C, Grignani G, Debernardi F, Regge D. Treatment of extraspinal painful bone metastases with percutaneous cementoplasty: a prospective study of 50 patients. Cardiovasc Intervent Radiol 2008: 31:1165-1173.

9. Toyota N, Naito A, Kakizawa H, et al. Radiofrequency ablation therapy combined with cementoplasty for painful bone metastases: initial experience. Cardiovasc Intervent Radiol 2005; 28:578-583.

10. Kawai $N$, Sato $M$, Iwamoto $T$, Tanihata $H$, Minamiguti $H$, Nakata $K$. Percutaneous osteoplasty with use of a cement-filled catheter for a pathologic fracture of the humerus. J Vasc Interv Radiol 2007; 18:805-809.

11. Nakata K, Kawai N, Sato M, et al. Percutaneous osteoplasty with a bone marrow nail for fractures of long bones: experimental study. J Vasc Interv Radiol 2010; 21:1436-1441.

12. Nakata K, Kawai N, Sato M, et al. Bone Marrow nails created by percutaneous osteoplasty for long bone fracture: comparisons among acrylic cement alone, acrylic-cement-filled bare metallic stent, and acrylic-cementfilled covered metallic stent. Cardiovasc Intervent Radiol 2010 (Epub ahead of print). 